\title{
Sustained co-delivery of BIO and IGF-I by a novel hybrid hydrogel system to stimulate endogenous cardiac repair in myocardial infarcted rat hearts
}

This article was published in the following Dove Press journal:

International Journal of Nanomedicine

28 July 2015

Number of times this article has been viewed

\begin{abstract}
Rui Fang, ',2 Shupei Qiao,' Yi Liu,' Qingyuan Meng, ${ }^{3}$ Xiongbiao Chen, ${ }^{2,4}$ Bing Song, ${ }^{5}$ Xiaolu Hou, ${ }^{6}$ Weiming Tian' 'Bio-X Center, School of Life Science and Technology, Harbin Institute of Technology, Harbin, People's Republic of China; 'Division of Biomedical Engineering, University of Saskatchewan, Saskatoon,

SK, Canada; ${ }^{3}$ State Key Laboratory of Molecular Developmental Biology, Institute of Genetics and Developmental Biology, Chinese Academy of Sciences, Beijing, People's Republic of China; ${ }^{4}$ Department of Mechanical Engineering, University of Saskatchewan, Saskatoon, SK, Canada; ${ }^{5}$ College of Biomedical and Life Sciences, Cardiff University, Cardiff, UK; ${ }^{6}$ Department of Cardiology, The Fourth Affiliated Hospital of Harbin Medical University, Harbin, People's Republic of China
\end{abstract}

Video abstract

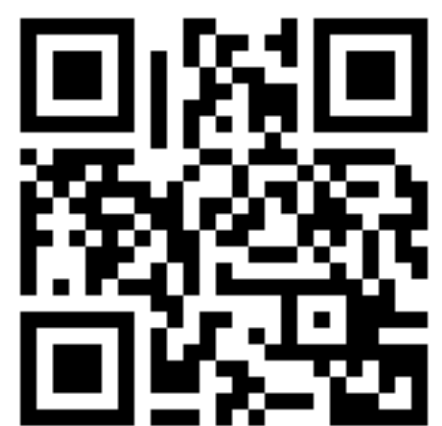

Point your SmartPhone at the code above. If you have a QR code reader the video abstract will appear. Or use: http://youtu.be/BFm9vImI7C4

Correspondence: Weiming Tian Bio-X Center, School of Life Science and Technology, Harbin Institute of Technology, No 2 Yikuang Street, Nangang District, Harbin 150080, People's Republic of China

Tel +8645 I 86403484

Fax +8645186403785

Email tianweiming@hit.edu.cn

Abstract: Dedifferentiation and proliferation of endogenous cardiomyocytes in situ can effectively improve cardiac repair following myocardial infarction (MI). 6-Bromoindirubin3-oxime (BIO) and insulin-like growth factor 1 (IGF-1) are two potent factors that promote cardiomyocyte survival and proliferation. However, their delivery for sustained release in MI-affected areas has proved to be challenging. In the current research, we present a study on the sustained co-delivery of BIO and IGF-1 in a hybrid hydrogel system to simulate endogenous cardiac repair in an MI rat model. Both BIO and IGF-1 were efficiently encapsulated in gelatin nanoparticles, which were later cross-linked with the oxidized alginate to form a novel hybrid hydrogel system. The in vivo results indicated that the hybrid system could enhance the proliferation of cardiomyocytes in situ and could promote revascularization around the MI sites, allowing improved cardiac function. Taken together, we concluded that the hybrid hydrogel system can co-deliver BIO and IGF-1 to areas of MI and thus improve cardiac function by promoting the proliferation of cardiomyocytes and revascularization.

Keywords: myocardial infarction, gelatin nanoparticle, cardiomyocyte proliferation, injectable hydrogel

\section{Introduction}

Many heart diseases primarily result in the loss of cardiomyocytes. It has been a significant challenge to develop effective treatments for cardiac repair because adult mammalian cardiomyocytes are highly differentiated cells and have long been thought to undergo terminal differentiation. ${ }^{1}$ Contrary to this long-held view, an emerging strategy posits that in response to heart injury, resident cardiomyocytes and cardiac stem cells surrounding the injured area can migrate and rapidly re-enter the cell cycle, thus promoting heart function recovery. ${ }^{2,3}$ However, the endogenous regenerative capacity of hearts is limited due to the massive loss of cardiomyocytes after myocardial infarction (MI) and heart failure. MI is considered a major cardiovascular disease, and it was found to be a major factor that contributed to non-natural mortality worldwide in $2013.4,5$

Current stem cell-based therapies have the potential to fundamentally improve the treatments of ischemic cardiac injury and heart failure. These therapeutic approaches mainly involve the use of bone marrow-derived mononuclear cells and their subsets, such as mesenchymal stem/stromal cells, endothelial progenitor cells, adipose tissuederived mesenchymal stem/stromal cells, cardiac tissue-derived stem cells, and cell combinations. ${ }^{6-9}$ However, these stem cell therapies have low efficacy due to poor cell engraftment and differentiation under the harsh (low nutrient and low oxygen) ischemic environment of infarcted hearts. ${ }^{6,7}$ Previous studies have shown that fewer than $2 \%$ of cells survive a few weeks after the stem cells' delivery. ${ }^{8-10}$ 
Despite the availability of many treatment options, heart disease remains the leading cause of death worldwide, raising the great need for novel or innovative therapeutic strategies. A recent study on stimulating cardiomyocyte dedifferentiation and proliferation by activating the mitotic signaling pathways involved in embryonic heart growth represents a complementary approach for heart regeneration and repair. ${ }^{11-14}$ An inhibitor of glycogen synthase kinase-3, 6-Bromoindirubin-3-oxime (BIO), which is isolated from mollusk Tyrian purple indirubins, has been shown able to induce the dedifferentiation of cardiomyocytes or endothelial cells, thus promoting mature cardiomyocyte proliferation..$^{15,16}$ On the other hand, substantial data also illustrate that insulinlike growth factor 1 (IGF-1) is a potent cardiomyocyte growth and survival factor. It has been illustrated that IGF-1 deletion has harmful effects on cardiac growth, and its deficiency is associated with an increased risk of cardiovascular disease. ${ }^{17,18}$ Cardiac-specific overexpression of IGF-1 can protect against myocyte apoptosis and ventricular dilation following MI. ${ }^{19,20}$

Recently, drug delivery systems using biomaterials as vehicles have been drawing considerable attention. Studies have shown that by combining growth factors with an injectable biomaterial, the biomaterial could serve as a controlled drug-release platform to improve functional outcomes..$^{21,22}$ However, co-delivery of BIO and IGF-1 to the injured heart area remains a challenge. Daily injections of both BIO and IGF-1 is a straightforward method that can maintain these agents at appropriate levels; however, this method causes issues, such as toxicity, due to their high doses. ${ }^{23}$ In the current research, we report a novel hybrid hydrogel system of chemically encapsulated gelatin nanoparticles (NPs) for the sustained co-delivery of BIO and IGF-1 in MI treatment.

\section{Materials and methods Materials}

Alginate (low viscosity), gelatin, sodium periodate, and hydrochloric acid were purchased from Sigma Aldrich Co (St Louis, MO, USA); BIO was purchased from EMD Millipore, a division of Merck KGaA (Darmstadt, Germany); IGF-1 was supplied by ProSpec-Tany TechnoGene Ltd (Rehovot, Israel; and Cy7 (molecular formula: $\mathrm{C}_{35} \mathrm{H}_{41} \mathrm{KN}_{2} \mathrm{O}_{8} \mathrm{~S}_{2}$ ) was purchased from Fanbo Biochemicals Co (Beijing, People's Republic of China).

\section{Experimental animals}

Sprague-Dawley (SD) male rats, specific pathogen free (SPF), (weighing 200-250 g each), provided by the Laboratory
Animal Center of Harbin Medical University (Harbin, People's Republic of China), were used to isolate cardiomyocytes, and they served as MI animal models. All animals were fed ad libitum and kept under the normal 12-hour dark/12-hour light cycle. All procedures were approved by the University Ethics Committee of the Harbin Institute of Technology.

\section{Preparation of blank gelatin NPs, BIO-loaded gelatin}

NPs, IGF-I-loaded gelatin NPs, and BIO-IGF-I co-loaded gelatin NPs

Blank gelatin NPs (BgNPs) were prepared using a two-step desolvation method with slight modifications. ${ }^{24,25}$ Briefly, gelatin was dissolved at $5 \mathrm{wt} \%$ in distilled water by stirring at $55^{\circ} \mathrm{C}$ for 2 hours. Then, the $\mathrm{pH}$ of the gelatin solution was adjusted to proceed with the second desolvation step. To prepare the BIO-loaded gelatin NPs (OgNPs), IGF-1-loaded gelatin NPs (FgNPs), and BIO-IGF-1 co-loaded gelatin NPs (CgNPs), $10 \mu \mathrm{L}$ of $5 \mu \mathrm{M}$ BIO (dissolved in $500 \mu \mathrm{L}$ of dimethyl sulfoxide [DMSO]) and $10 \mu \mathrm{L}$ of $10 \mathrm{mM}$ IGF-1 were added individually or together into $10 \mathrm{~mL}$ of $5 \%$ gelatin solution in a dark environment, respectively, followed by the dropwise addition of acetone $(30 \mathrm{~mL})$ to form NPs. At the end of the process, a glutaraldehyde solution $(25 \% \mathrm{v} / \mathrm{v}$ aqueous solution) was added as a cross-linking agent, and the solution was stirred for 12 hours at a speed of $600 \mathrm{rpm}$. DMSO was removed by means of distilled water. Eventually, the solution was centrifuged at 12,000 rpm for 1.5 hours, yielding the NPs. Dynamic light scattering was conducted using a Zetasizer Nano ZS system (Malvern Instruments, Malvern, UK) to characterize the distribution and size of the NPs.

\section{Methods}

\section{Encapsulation efficiencies of NPs}

The encapsulation efficiency of the BIO and IGF-1 within the NPs was evaluated using an ultraviolet (UV) method. A drug-encapsulating gelatin NP solution was filtered through a Millipore filter (UFP2THK24 [100 kD cutoff]), where the free drug present in the aqueous buffer passed through the filter, leaving the drug entrapped in the NPs. Following the separation of the NPs from the aqueous buffer, the drug left in the aqueous buffer was measured using a $752 \mathrm{UV} /$ visible recording spectrophotometer with a wavelength of $500 \mathrm{~nm}$ and $280 \mathrm{~nm}$, respectively. The total amount of drug left in the aqueous solution was subtracted from the amount of drug originally added in the reaction medium, and the entrapment efficiency (E \%) was calculated from the ratio of the amount of drug entrapped over the total amount of drug added. 
Synthesis of self-cross-linked hydrogel and its physicochemical characterization

Hydrogel was synthesized as previously described. ${ }^{26}$ Briefly, oxidized alginate was obtained by mixing sodium peroxide and sodium alginate (in distilled water) with mass ratios of 1:2. The reaction was conducted at $4^{\circ} \mathrm{C}$ overnight, and the degree of oxidation was evaluated by measuring the concentration of sodium peroxide, which was left unconsumed after 24 hours. The oxidized alginate and gelatin hydrogel were then obtained by mixing the alginate dialdehyde and gelatin NP solutions. The gelation process and the mechanical properties of oxidized alginate and gelatin hydrogel were evaluated by examining the time of gelation onset and the evolution of elasticity at $37^{\circ} \mathrm{C}$ in the constant strain mode by means of a Bohlin Gemini II rheometer (Malvern Instruments) featuring a parallel plate geometry $(40 \mathrm{~mm}$ in diameter), as in our previous study. ${ }^{26}$

\section{Drug-releasing profile in vitro and in vivo}

To monitor the in vitro release profile, gelatin NPs and each of the encapsulating drugs were made to conform by means of a semi-permeable membrane $(40 \mathrm{kD})$ and were then immersed in $10 \mathrm{~mL}$ of phosphate buffered saline at $4{ }^{\circ} \mathrm{C}$ for release within a time period of 7 days; every 24 hours, the optical density was examined by a UV spectrophotometer at $500 \mathrm{~nm}$. A sample of $1 \mathrm{~cm}^{3}$ of the encapsulating drug $(10 \mu \mathrm{L})$ was examined under the same conditions as the control group, so as to study the drug's release profile.

To monitor the in vivo release profile, an in vivo imaging method was used. First, the gelatin NPs were labeled with the fluorescent agent $\mathrm{Cy} 7$, and then the labeled gelatin NPs were conjugated to the hydrogel, as described in the "Synthesis of self-cross-linked hydrogel and its physicochemical characterization" section above. Both the labeled gelatin NPs alone, as well as the labeled gelatin NPs that conjugated to the hydrogels, were injected into the area affected by MI in 20 rats (ten rats in each group), as described in a previous study. ${ }^{26}$ The fluorescence of both groups was examined and recorded by an in vivo imaging system (DXS 4000 PRO; Eastman Kodak Co, Rochester, NY, USA) every 5 days.

\section{Cardiomyocyte culture}

Neonatal cardiomyocytes were isolated from 2- to 3-day-old SD rats, as adapted from a previous protocol. ${ }^{27}$ Hearts were removed from the neonatal rats; then, atrial and connective tissues were excised. Hearts were minced into $1 \mathrm{~mm}^{3}$ pieces and then were subjected to five rounds of enzymatic digestion for $8-10$ minutes with collagenase II $(0.5 \mathrm{mg} / \mathrm{mL}$; Worthington
Biochemical Corporation, Lakewood, NJ, USA). Digestion was stopped with an equal volume of Dulbecco's Modified Eagle's Medium (DMEM) containing 10\% fetal bovine serum (FBS) (Thermo Fisher Scientific, Waltham, MA, USA). The cell suspension was centrifuged for 5 minutes at $2,000 \times g$, and the resulting pellet was resuspended in DMEM containing $10 \%$ FBS. Cells were pre-plated in cell culture flasks to reduce non-myocyte contamination (Corning Incorporated, Corning, NY, USA) for 90 minutes. Isolated cardiomyocytes were made into a suspension using oxidized alginate conjugated with gelatin NPs at a density of 105 cells $/ \mathrm{mL}$. The purity of the cardiomyocyte suspension was evaluated by immunofluorescence staining with an anti-sarcomeric antibody.

\section{Cell proliferation in hydrogels assay}

After 7 days of culture, the hydrogel/cardiomyocyte constructs were fixed. Immunofluorescence was performed with the proliferating cell nuclear antigen (PCNA) antibody (1:200; Abcam plc, Cambridge, UK) to identify the proliferation of cardiomyocytes. Laser confocal images were observed, and the PCNA-positive cells were counted.

\section{MI animal model preparation and hydrogel implantation}

Eighty-five male SD rats (weighing $250 \mathrm{~g}$ each) were operated on to create the MI animal models. The rats were anesthetized with an intravenous injection of 3\% pentobarbital sodium (30 $\mathrm{mg} / \mathrm{kg}$ ), and an MI was subsequently induced by ligating the left anterior descending coronary artery $2 \mathrm{~mm}$ from the top of the normally positioned left auricle. One week later, upon confirmation of an induced MI by means of echocardiography using a cardiovascular ultrasound system (VIVID-7; GE Healthcare Bio-Sciences Corp, Piscataway, NJ, USA), each rat was injected with $80 \mu \mathrm{L}$ of a given hydrogel (Table 1) into the anterior and lateral regions bordering the infarct, as well as at the center

Table I Groups and number of animals used for in vivo MI repair

\begin{tabular}{lll}
\hline Groups & $\begin{array}{l}\text { Number of animals } \\
\text { per group }\end{array}$ & $\begin{array}{l}\text { Number of animals } \\
\text { survived at 6 weeks } \\
\text { post-surgery }\end{array}$ \\
\hline Sham & 15 & 12 \\
Control & 20 & 11 \\
IGF-I & 20 & 14 \\
BIO & 20 & 17 \\
BIO and IGF-I & 20 & 16 \\
\hline
\end{tabular}

Abbreviations: MI, myocardial infarction; BIO, 6-bromoindirubin-3-oxime; IGF-I, insulin-like growth factor $\mathrm{I}$. 
of the infarct ( $16 \mu \mathrm{L}$ per region). At 6 weeks post-injection, echocardiography was performed on the anesthetized rats. Specifically, two-dimensional (2D) images were captured at the mid-papillary muscle level, and using M-mode tracings, the left ventricular (LV) ejection fraction (LVEF), fractional shortening (FS), the LV end systolic diameter (LVSD), and the LV end diastolic diameter (LVDD) were evaluated from at least three consecutive cardiac cycles.

\section{Histology and immunohistochemistry}

At 6 weeks post-implantation, the rat hearts were arrested with $10 \% \mathrm{KCl}$, rapidly excised, and frozen in tissue medium, which was followed by sectioning each heart into three or four transverse slices parallel to the atrioventricular ring. Samples were then fixed with $10 \%$ buffered formalin, embedded in paraffin, sectioned into $5 \mu \mathrm{m}$ slices, and stained with hematoxylin and eosin (H\&E). Scar thickness was measured under a microscope, and on this basis, the relative scar thickness (defined by the average scar thickness divided by the average wall thickness) and the heart expansion index (defined by the LV cavity area/whole LV area/relative scar thickness) were evaluated, as in our previous study. ${ }^{26}$

The detection of various tissue antigens was conducted using the primary antibody, CD31, for endothelial cells (Abcam plc). Double staining with anti-cardiac troponin-T (green, cardiomyocyte marker; Abcam plc) and anti-PCNA (red, proliferation marker; Abcam plc) was performed. The doubled-stained cells were evaluated by counting the average number of positively stained cells within six areas of $0.1 \mathrm{~mm}^{2}$. The CD31-positive capillary densities at the infarcted areas were analyzed by employing the same method.

\section{Statistical analysis}

All of the results were reported as the mean \pm standard deviation. Two-way analysis of variance (ANOVA) was used in the analysis of heart function between groups, and Student's $t$-test was used for the remainder of the analysis. A $P$-value $<0.05$ was considered significant; a $P$-value $<0.01$ was considered very significant.

\section{Results}

Preparation and characterization of hydrogels and the drug-release profile in vitro and in vivo

The morphology of BgNPs was observed using a scanning electron microscope (SEM) (Figure 1A); the results of dynamic light scattering showed that the NPs formed a cluster featuring different diameters ranging from $180 \mathrm{~nm}$ to $255 \mathrm{~nm}$ with a polydispersity index (PDI) of $0.158 \pm 0.029$ (Figure 1B).
Then, the NPs were covalently attached to the hydrogel, as we have previously reported. ${ }^{24}$ The hydrogel formed with the porous network structure (Figure 1C). The loading efficiency of OgNPs and FgNPs, which contained BIO and IGF-1, respectively, were about $55 \%$ or $60 \%$, which is similar to the findings of a previous study. ${ }^{28}$ To detect the release profile of the NPs and the hydrogel composite system, we designed a method to examine the BIO release profile in the NPs conjugated to the hydrogel and in the free NPs, as described in the encapsulation efficiencies of NPs section. We compared the release behavior of BIO in NPs and in NPs covalently conjugated to the hydrogel.

The release profile assay lasted for 110 hours for both groups. The release rate of BIO in NPs was much higher than the release rate of BIO in NPs encapsulated in hydrogel (Figure 1D). From 0 to 60 hours, a rapid increase in the drug release was observed in the NPs; however, a similar release peak was detected from 60 to 90 hours in the NPs conjugated to the hydrogel (Figure 1D). For NPs, the BIO release mechanisms included the biodegradation of NPs; in addition, BIO diffused to the solution outside the semipermeable membrane.

In contrast with NPs, two steps were involved in the BIO's release profile in NPs covalently conjugated to the hydrogel. The first step involved the breakage of amines found between the NPs and the hydrogel, and the second step included BIO's release from the NPs. As a result, the peak release was postponed 60 hours in the in vitro delivery system. To further confirm the release mechanism of NPs, the mechanical properties of the hydrogel were measured and compared to its original properties prior to being released. The results showed that the elastic and viscous moduli of the hydrogel were around 105 and $104 \mathrm{~Pa}$ prior to release, respectively, and they had decreased to 103 and $10 \mathrm{~Pa}$, respectively, after release (Figure 1E).

However, there was a tendency for the elastic and viscous moduli to stay the same before and after the release, which indicated that the hydrogel still maintained a gel network structure. Therefore, we postulated that the main releasing mechanism occurs through the breakage of chemical bonds between gelatin NPs and oxide alginate-gelatin hydrogel. The release profile was also controlled by the cross-linking density and degradability of the gel, modulated by the initial gelation condition. The in vivo release profile was evaluated using the in vivo imaging method, as shown in Figure 2A. The results illustrated that the fluorescence of the scaffold encapsulating Cy7-labeled NPs could last for 45 days, while the fluorescence of the control group (free Cy7-labeled NPs, 
A

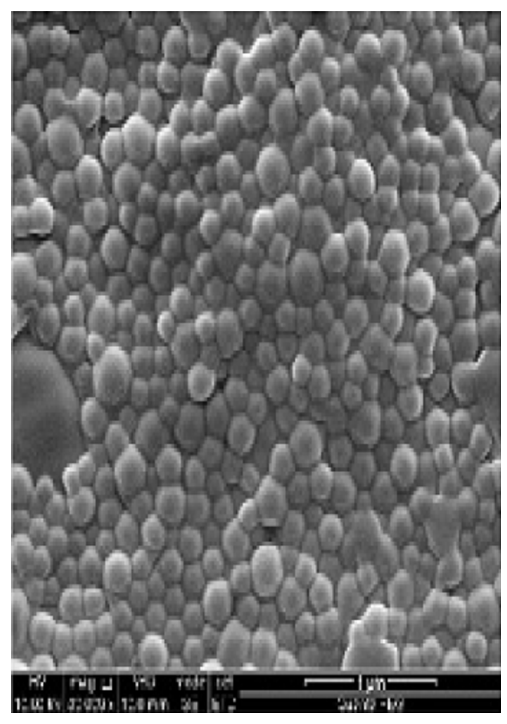

B

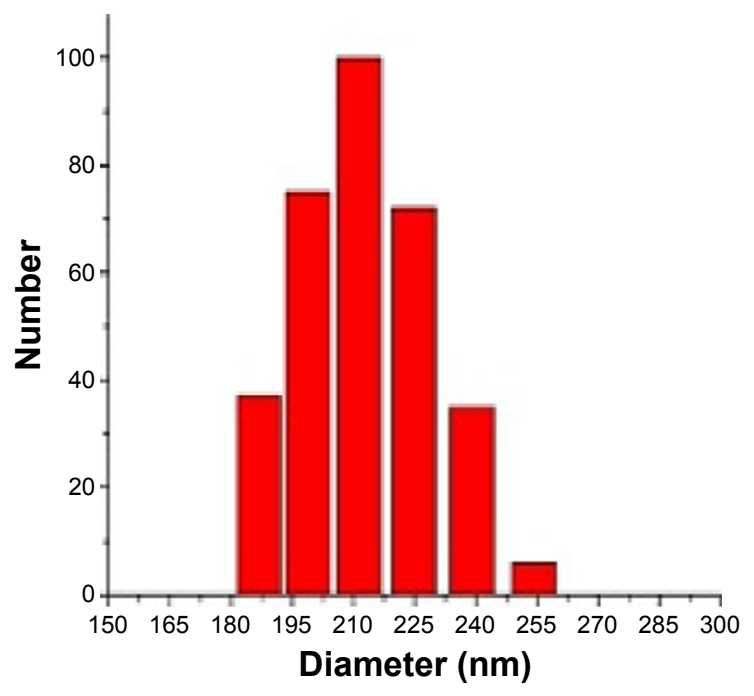

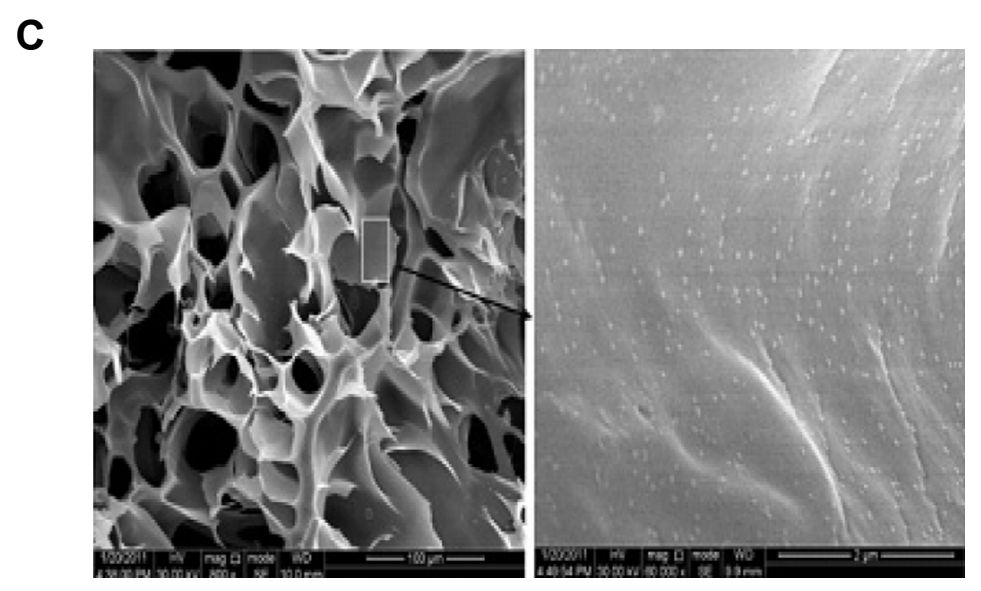

D

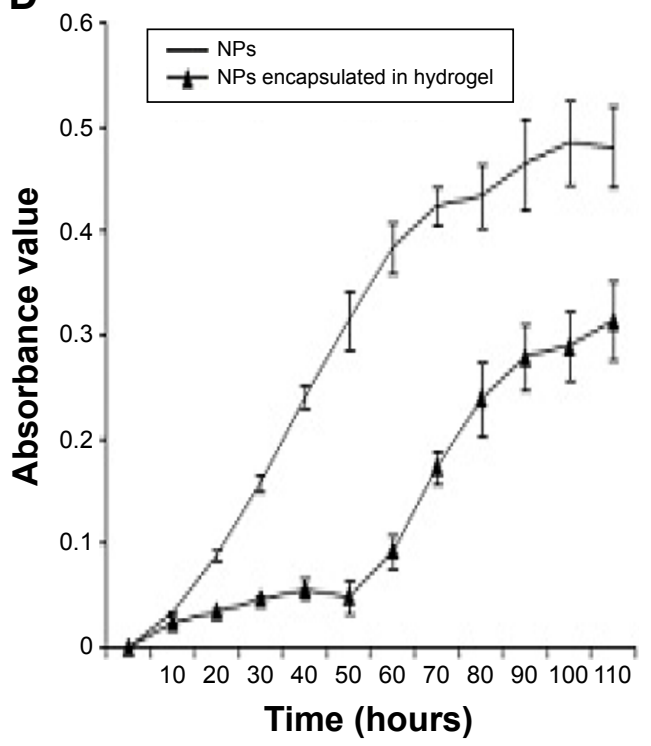

E

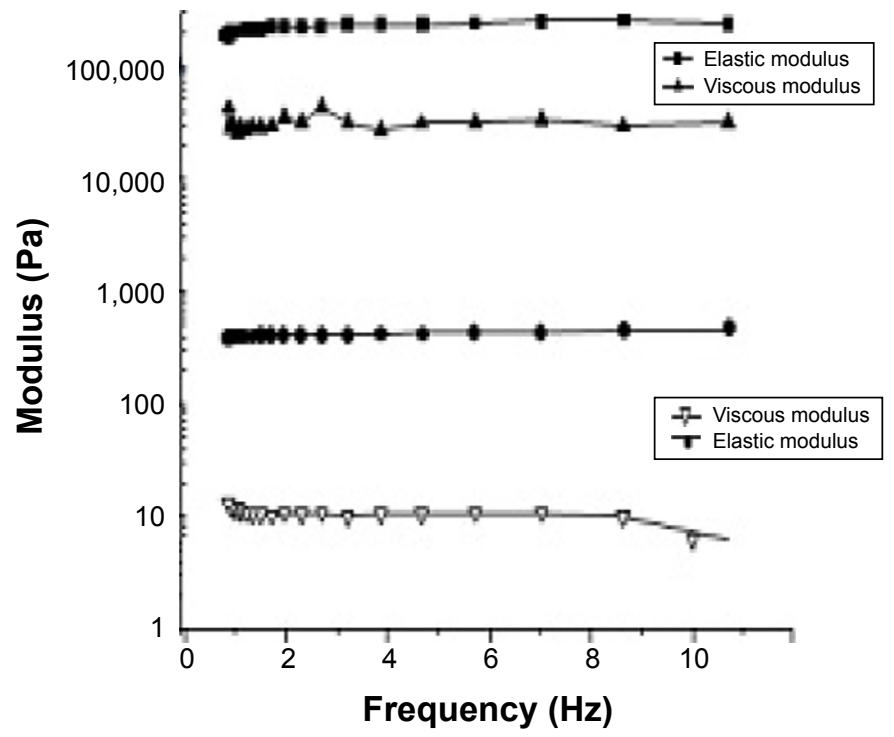

Figure I Release assay profile in vitro.

Notes: (A) SEM morphology of the prepared gelatin NPs. (B) DLS analysis of the gelatin NPs. (C) SEM image and a zoomed image showing the gelatin NPs covalently conjugated to the hydrogel. (D) The UV results of BIO absorption showing the release profiles in the free NP group and in the gelatin NP encapsulated in hydrogel group. (E) Mechanical properties of the hydrogel before and after release.

Abbreviations: SEM, scanning electron microscope; DLS, dynamic light scattering; BIO, 6-bromoindirubin-3-oxime; NP, nanoparticle; UV, ultraviolet. 
A

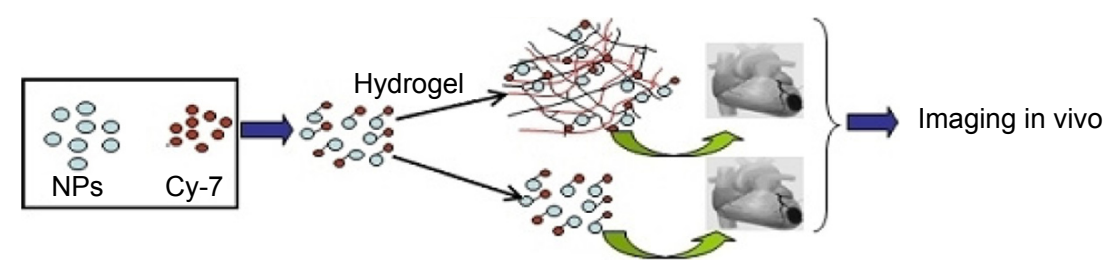

\section{B}
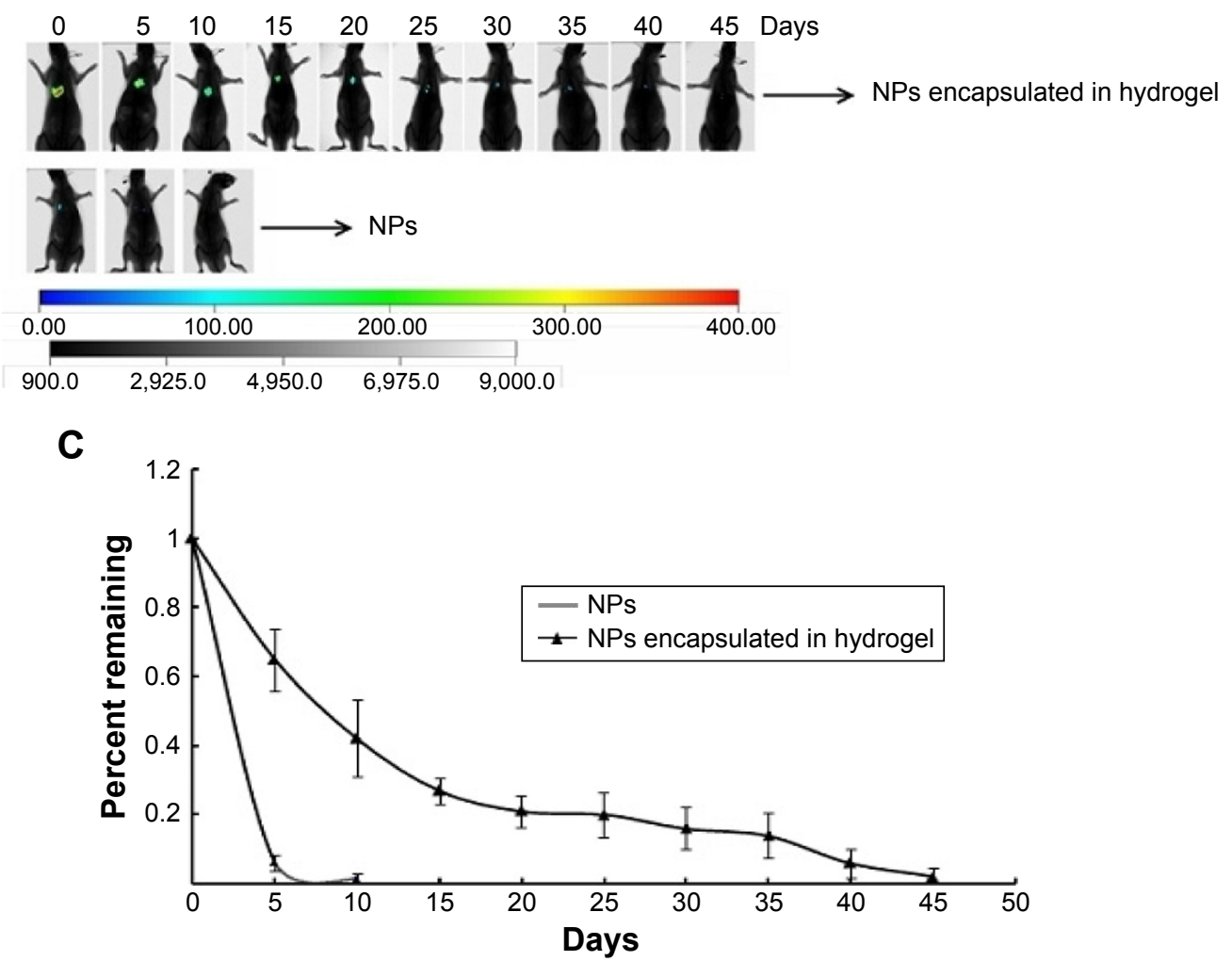

Figure 2 In vivo release profile assay.

Notes: (A) The scheme showing the strategy used to monitor the release profile using an in vivo imaging method. (B) Typical images of rats implanted with free Cy7-labeled NPs and Cy7-labeled gelatin NPs encapsulated in hydrogel. $(\mathbf{C})$ The statistical analysis of immunofluorescence intensity remained constant over time ( $\mathrm{n}=6$ ).

Abbreviation: NP, nanoparticle.

which were not conjugated to the hydrogel) lasted for less than 10 days (Figure 2B, C). Moreover, the intensities of the fluorescence were much higher than those in the control group (Figure 2C).

\section{Cardiomyocyte proliferation detected in hydrogels}

To mimic the in vivo effect of the drug delivery system among cardiac cells, a three-dimensional (3D) system was used to encapsulate the cardiomyocytes in hydrogel in the present study. The results showed that the percentage of proliferated cardiomyocytes in the BIO release system $(15 \% \pm 4 \%)$ was significantly higher than that in the control groups $(7 \% \pm 3 \%$; $P<0.05)$; however, no significant difference was observed in the IGF-1 release group $(11 \% \pm 2 \%)$, as shown by the laser confocal images (Figure 3A-C) and the statistical analyses (Figure 3D). Therefore, IGF-1 could not induce the proliferation of cardiomyocytes.
BIO/IGF-I administration improves cardiac function after $\mathrm{MI}$ and leads to the formation of new cardiomyocytes

MI animal models were prepared according to the method described above, and different hydrogels that covalently conjugated with various NPs (blank gelatin NPs, OgNPs, FgNPs, and CgNPs) were implanted to detect their effects on cardiac function repair. With echocardiography, the LVEF, FS, LVDD, and LVSD of the hearts in the different groups were obtained, and the results are listed in Table 2. It can be observed that after 6 weeks of treatment, the LVEF in the control group decreased significantly from $28.2 \pm 5.8$ to $18.8 \pm 3.5$, which suggested that the heart progressed to myocardial dysfunction. In contrast, the LVEF in animals treated with BIO or IGF-1, and those treated with the co-delivery of both BIO and IGF-1, showed a significant increase. Compared with the control group at 6 weeks post-injection (18.8 \pm 3.5$)$, 


\section{A}

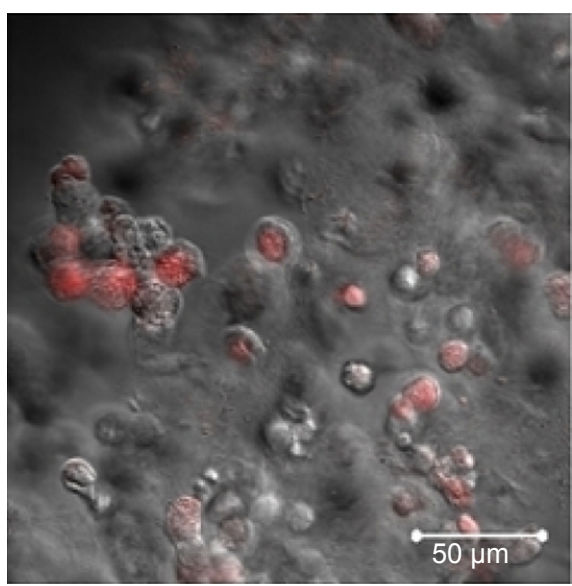

C

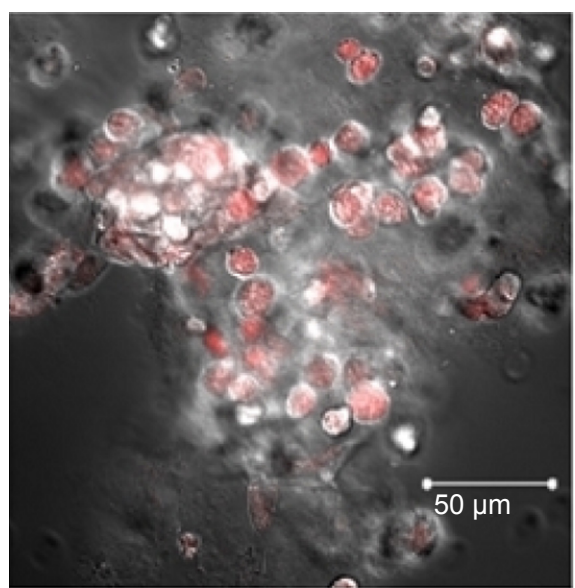

B

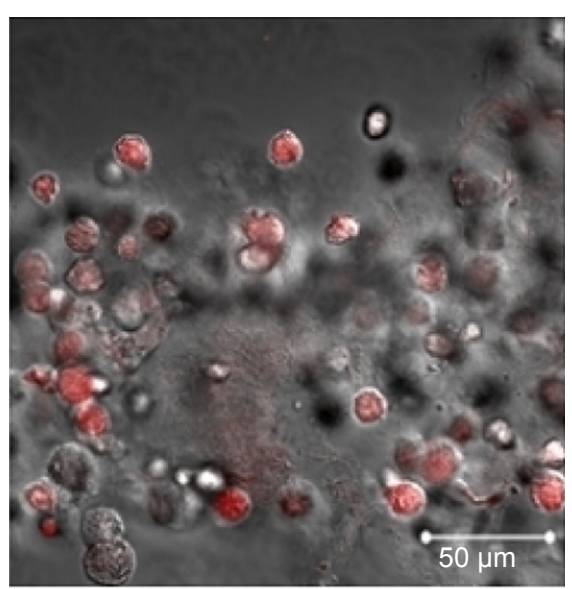

D

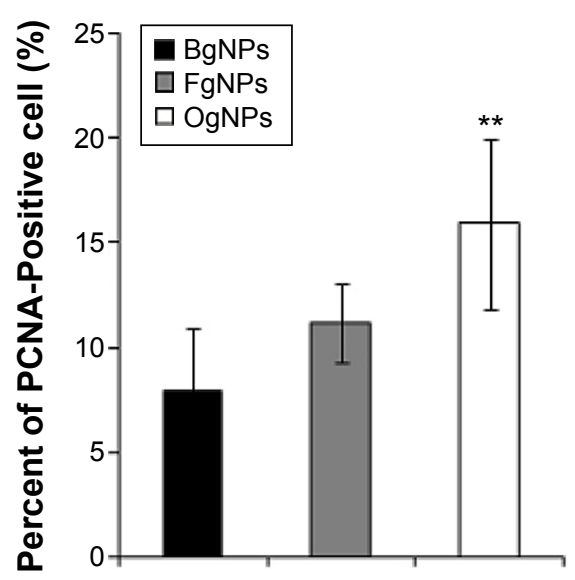

Figure 3 Effect of the hydrogen delivery system on the proliferation of 3D culture cardiomyocytes.

Notes: Scheme showing the strategy used to detect the enhanced proliferation of 3D-cultured cardiomyocytes. Laser confocal images showing the PCNA-positive stained

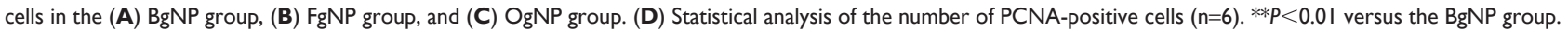
Abbreviations: BIO, 6-bromoindirubin-3-oxime; 3D, three dimensional; PCNA, proliferating cell nuclear antigen; NP, nanoparticle; BgNP, blank gelatin nanoparticle; OgNP, BIO-loaded gelatin nanoparticle; IGF-I, insulin-like growth factor I; FgNP, IGF-I-loaded gelatin nanoparticle.

Table 2 Functional evaluation of ischemic heart

\begin{tabular}{lllll}
\hline & LVEF & FS & LVDD (cm) & LVSD (cm) \\
\hline $\begin{array}{c}\text { Sham } \\
\text { Baseline }\end{array}$ & $56.0 \pm 8.7$ & $30.5 \pm 2.0$ & $0.66 \pm 0.02$ & $0.48 \pm 0.01$ \\
$\quad$ Week 6 & $53.5 \pm 7.8$ & $31.3 \pm 2.3$ & $0.67 \pm 0.02$ & $0.50 \pm 0.02$ \\
Control & & & & \\
$\quad$ Baseline & $28.2 \pm 5.8$ & $12.3 \pm 1.9$ & $0.78 \pm 0.08$ & $0.42 \pm 0.03$ \\
Week 6 & $18.8 \pm 3.5$ & $10.3 \pm 2.1$ & $0.95 \pm 0.09$ & $0.65 \pm 0.06$ \\
IGF-I & & & & \\
$\quad$ Baseline & $29.1 \pm 5.5$ & $12.9 \pm 1.8$ & $0.76 \pm 0.05$ & $0.44 \pm 0.03$ \\
$\quad$ Week 6 & $42.8 \pm 6.2^{*}$ & $17.7 \pm 2.3^{*}$ & $0.81 \pm 0.09 *$ & $0.38 \pm 0.02^{*}$ \\
BIO & & & & \\
Baseline & $27.5 \pm 5.9$ & $12.6 \pm 2.0$ & $0.77 \pm 0.09$ & $0.40 \pm 0.03$ \\
$\quad$ Week 6 & $32.1 \pm 4.3^{*}$ & $14.3 \pm 2.2^{*}$ & $0.75 \pm 0.04^{*}$ & $0.39 \pm 0.02^{*}$ \\
BIO and IGF-I & & & & \\
Baseline & $28.6 \pm 4.9$ & $11.8 \pm 1.7$ & $0.79 \pm 0.08$ & $0.45 \pm 0.04$ \\
Week 6 & $55.6 \pm 8.7^{*}$ & $27.4 \pm 3.8^{*}$ & $0.82 \pm 0.09^{*}$ & $0.39 \pm 0.02^{*}$ \\
\hline
\end{tabular}

Notes: *The $P$-value of $<0.05$ was considered significant from their specific control group. Data are expressed as the mean \pm standard error of the mean.

Abbreviations: BIO, 6-bromoindirubin-3-oxime; IGF-I, insulin-like growth factor I; LVEF, left ventricular ejection fraction; FS, fractional shortening; LVDD, left ventricular end diastolic diameter; LVSD, left ventricular end systolic diameter. significant improvements in the LVEF of animals treated with BIO (32.1 $\pm 4.3 ; P<0.05)$, IGF-1 (42.8 $\pm 6.2 ; P<0.05)$, and the co-delivery of both BIO and IGF-1 (55.6 \pm 8.7 ; $P<0.01)$ were found. At 6 weeks post-injection, the FS\% slightly decreased in the BIO group; however, the FS\% had increased significantly in both the BIO and IGF-1 groups when compared with baseline. It was also higher in the BIO plus IGF-1 (BIO + IGF-1) group than in the BIO or control groups (Table 2).

In order to study the mechanism underlying the advancement of cardiac function for the administration of $\mathrm{BIO}+$ IGF-1 in the MI rats, H\&E staining was used perform a histology evaluation following injection with our hydrogel system. Cardiac remodeling was found in our experimental groups; however, when compared with the control group, more cardiomyocytes were found in the BIO + IGF-1 group (Figure 4), and the results were further confirmed by double staining with 

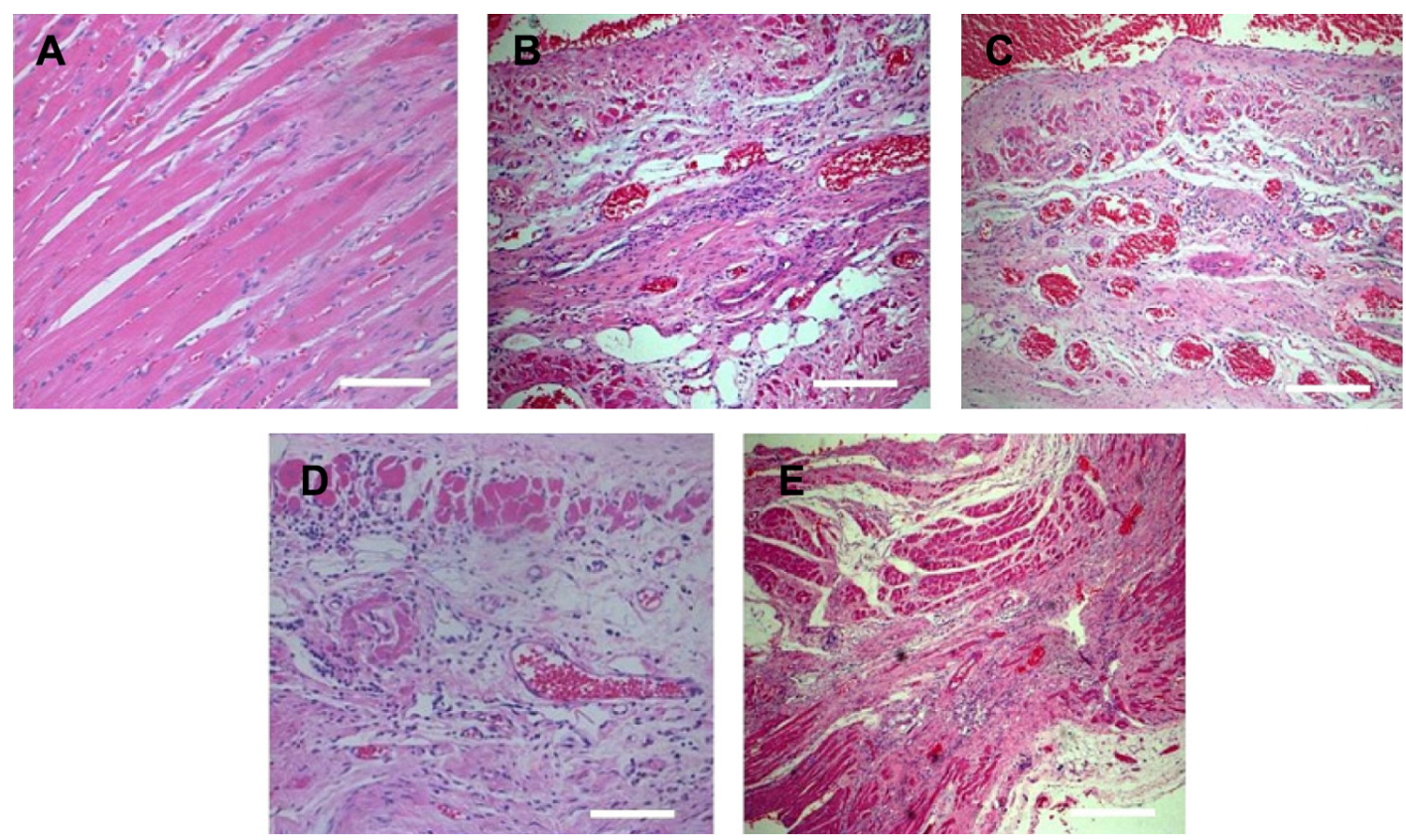

Figure 4 Histology evaluation of an ischemic heart.

Notes: (A-E) H\&E staining of the tissue sections obtained from the MI region among the (A) sham group, (B) control group, (C) IGF-I release group, (D) BIO release group, and (E) BIO and IGF-I co-delivery group. Scale bar, $100 \mu \mathrm{m}$.

Abbreviations: BIO, 6-bromoindirubin-3-oxime; IGF-I, insulin-like growth factor I; H\&E, hematoxylin and eosin; MI, myocardial infarction.

the anti-cardiac troponin- $\mathrm{T}$ (green; cardiomyocyte marker) and anti-PCNA (red; proliferation marker) assay. The number of proliferating cardiomyocytes in the BIO + IGF-1 group (35 5), was the most significant among all groups, and the number of proliferating cardiomyocytes in the BIO group $(27 \pm 5)$ was also significantly higher than that of both the

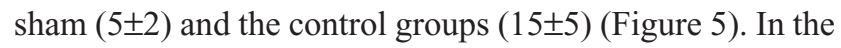
BIO delivery group, the enhanced proliferation of cardiomyocytes was observed. In the co-delivery of the BIO + IGF-1 group, both enhanced proliferation of the cardiomyocytes and function recovery were seen. Moreover, angiogenesis plays a crucial role in tissue repair and heart function after MI; therefore, the CD31 antibody-positive angiogenesis assay was detected among the different groups (Figure 6). In the BIO + IGF-1 group, the most capillaries $\left(70 \pm 8 / \mathrm{mm}^{2}\right)$ was observed. In addition, significant increase of blood vessels $\left(60 \pm 7 / \mathrm{mm}^{2}\right)$ in the IGF-1 group was also detected. However, no significant increase in blood vessel density was observed in the BIO group $\left(43 \pm 8 / \mathrm{mm}^{2}\right)$, which suggested that BIO could not promote angiogenesis. Enhanced angiogenesis was accompanied by functional recovery in the BIO and IGF-1 group; this indicates that angiogenesis may be an important factor in the recovery of function.

\section{Discussion}

In the present study, we investigated the functional and histological/cellular effects of the intramyocardial administration of BIO and IGF-1 in MI rats. We showed that the improved sustained co-delivery of BIO and IGF-1 by a newly developed hybrid hydrogel system holds potential as a novel treatment for MI. Six weeks after delivery, BIO and IGF-1 treatment led to the proliferation of resident cardiac cells and promoted revascularization. Importantly, the repair and regeneration of the damaged myocardial tissues was associated with significant improvements in cardiac function (Figure 7).

\section{Development of a new hybrid hydrogel system}

Recently, studies have reported on the ways in which cardiac diseases can be treated through the sustained delivery of IGF-1 by means of hydrogels. ${ }^{29,30}$ Though it makes a difference in the treatment of MI, IGF-1 also causes such issues as toxicity due to their high doses and the short release profile. Gelatin NPs, due to their good biocompatibility and biodegradability, have been widely utilized in drug delivery applications. ${ }^{31}$ However, the relatively fast degradation of gelatin limits its applications to long-term protein delivery. Our previous study showed that the covalent cross-linking of gelatin with alginate forms a hydrogel with both enhanced mechanical properties and bioactive motifs for cell attachment, biodegradability, and functional recovery. ${ }^{26}$ In the current study, we developed a novel hybrid hydrogel system with chemically encapsulated gelatin NPs, which are 

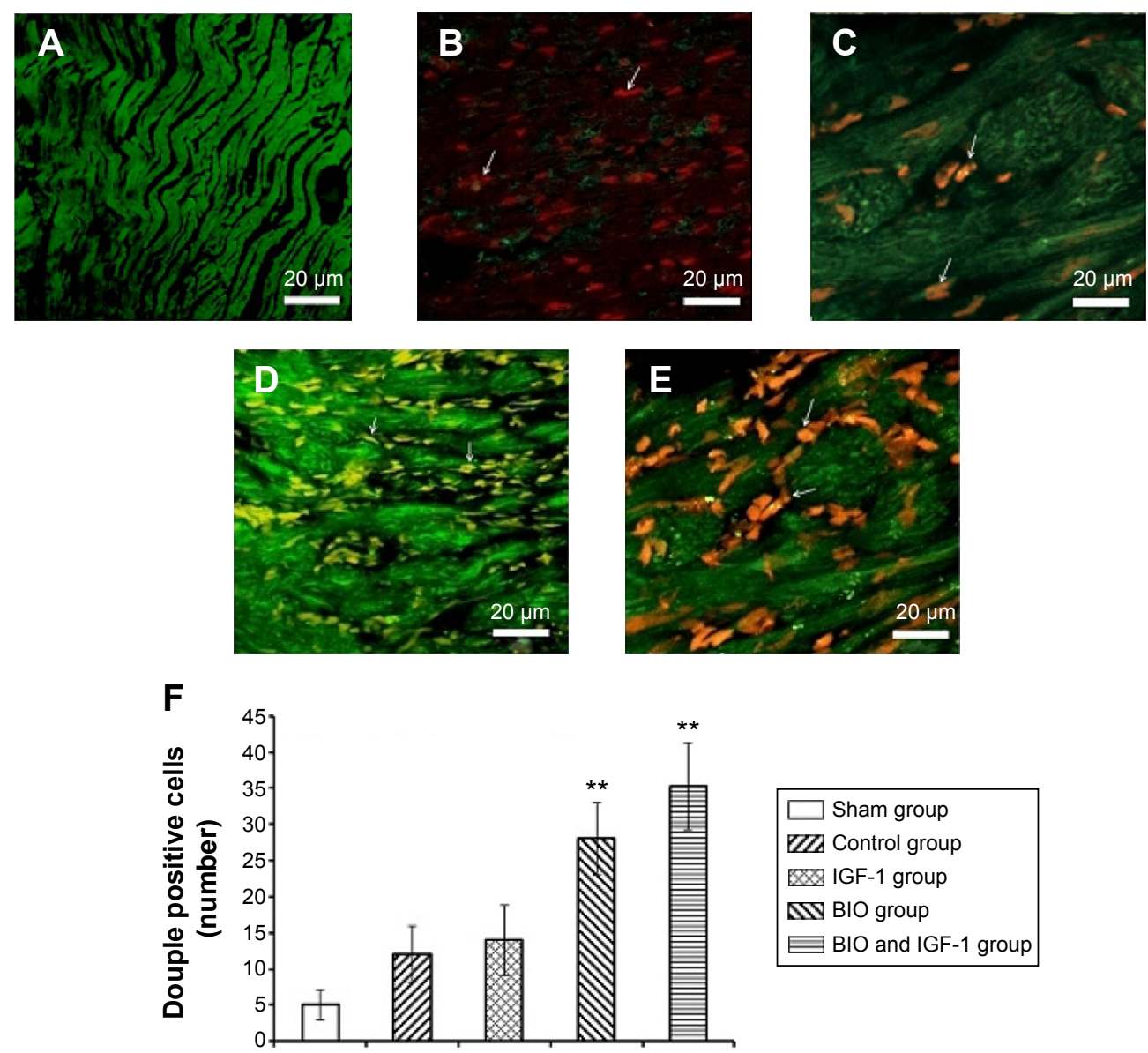

Figure 5 Double staining with anticardiac troponin-T (green; cardiomyocyte marker) and anti-PCNA (red; proliferation marker) shows the enhanced proliferation of resident cardiomyocytes in an $\mathrm{MI}$ model $(P<0.0 \mathrm{I} ; \mathrm{n}=6)$.

Notes: (A) Sham group, (B) control group, (C) IGF-I release group, (D) BIO release group, and (E) BIO and IGF-I co-delivery group. (F) Statistical analysis of the number of double-stained cells. ${ }^{* * P}<0.01$ versus the control group. The arrows show the cardiomyocytes which are double staining.

Abbreviations: BIO, 6-bromoindirubin-3-oxime; IGF-I, insulin-like growth factor I; MI, myocardial infarction; PCNA, proliferating cell nuclear antigen.

$\mathrm{pH}$ sensitive, for the sustained co-delivery of BIO + IGF-1 in MI treatment. The oxidation of sodium alginate produced aldehyde groups, which can form unstable amines found within the free amino group in the gelatin NPs; thus, they covalently linked the NPs to the alginate polymer chain. An acidic, low-pH environment was generated with the occurrence of an MI. ${ }^{5}$ The amines that were found, which form between alginate and the gelatin NPs, are most active in acidic environments. Following MI, the bond broke down quickly, and the BIO and IGF-1 were released to the damaged area of the heart, ultimately promoting the heart's overall function.

In summary, in our current study, we developed a novel, pH-sensitive, hybrid hydrogel system of chemically encapsulated gelatin NPs that can more efficiently sustain the co-delivery of BIO and IGF-1 to the injured area of the heart. Further, we also illustrated that the system could be more advantageous when delivering BIO and IGF-1 in the treatment of MI.

\section{Sustained release of BIO or IGF-I using hybrid hydrogel systems}

Drug release profiles were detected both in vitro and in vivo. It is generally recognized that $2 \mathrm{D}$ cell cultures are not adequate representatives of the cellular environment found in organisms; rather, 3D cell culture models have the potential to improve the physiological relevance of cell-based assays. ${ }^{32}$ In our previous study, we constructed an engineered heart tissue model using cardiomyocytes. ${ }^{26}$ In order to mimic the in vivo effect of a drug delivery system when promoting the proliferation of cardiac cells, a 3D system to encapsulate the cardiomyocytes in the hydrogels was used in the present study.

The drug release rate was detected in vivo by an imaging system that has been widely used in cancer studies. More recently, a study showed that it can also be used to evaluate biomaterial biodegradation in vivo. ${ }^{33} \mathrm{Cy} 7$ was conjugated to the NPs, and the rapidly decreasing fluorescence intensities in the NP group indicated that without the support of 

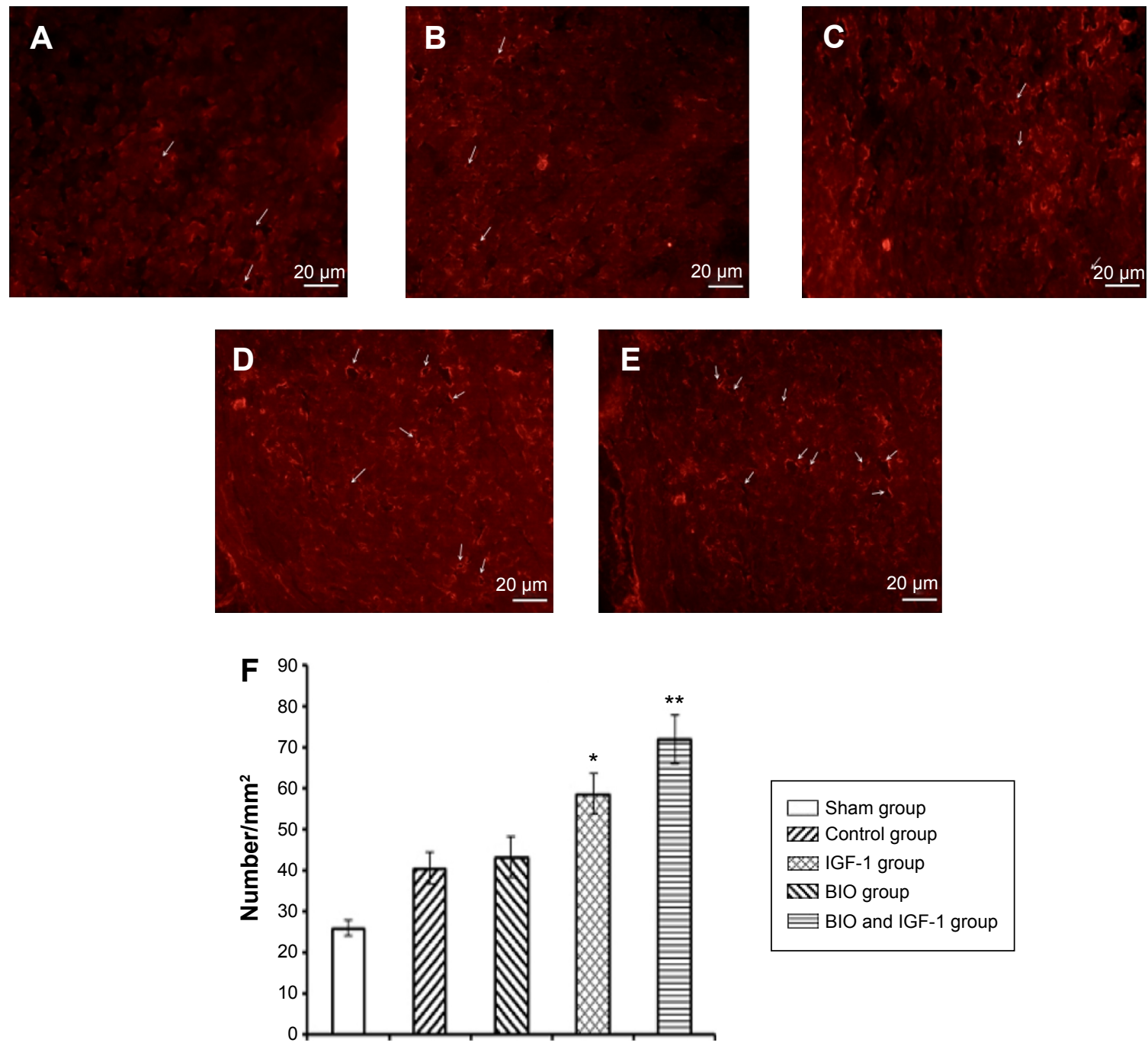

\section{$\square$ Sham group}

ZZI Control group

$\bowtie$ IGF-1 group

NI BIO group

Е BIO and IGF-1 group

Figure 6 Immunostaining for the evaluation of angiogenesis.

Notes: (A-D) CD3I immunostaining of the tissue sections from the MI among the (A) sham group, (B) control group, (C) IGF-I release group, (D) BIO release group, and (E) BIO and IGF-I co-delivery group. (F) Statistical analysis of the CD3I-positive blood vessels $(n=6)$. $* P \leq 0.05$ versus the control group; $* * P<0.0$ I versus the control group. The arrows point to the endothelial cells.

Abbreviations: BIO, 6-bromoindirubin-3-oxime; IGF-I, insulin-like growth factor I; MI, myocardial infarction.

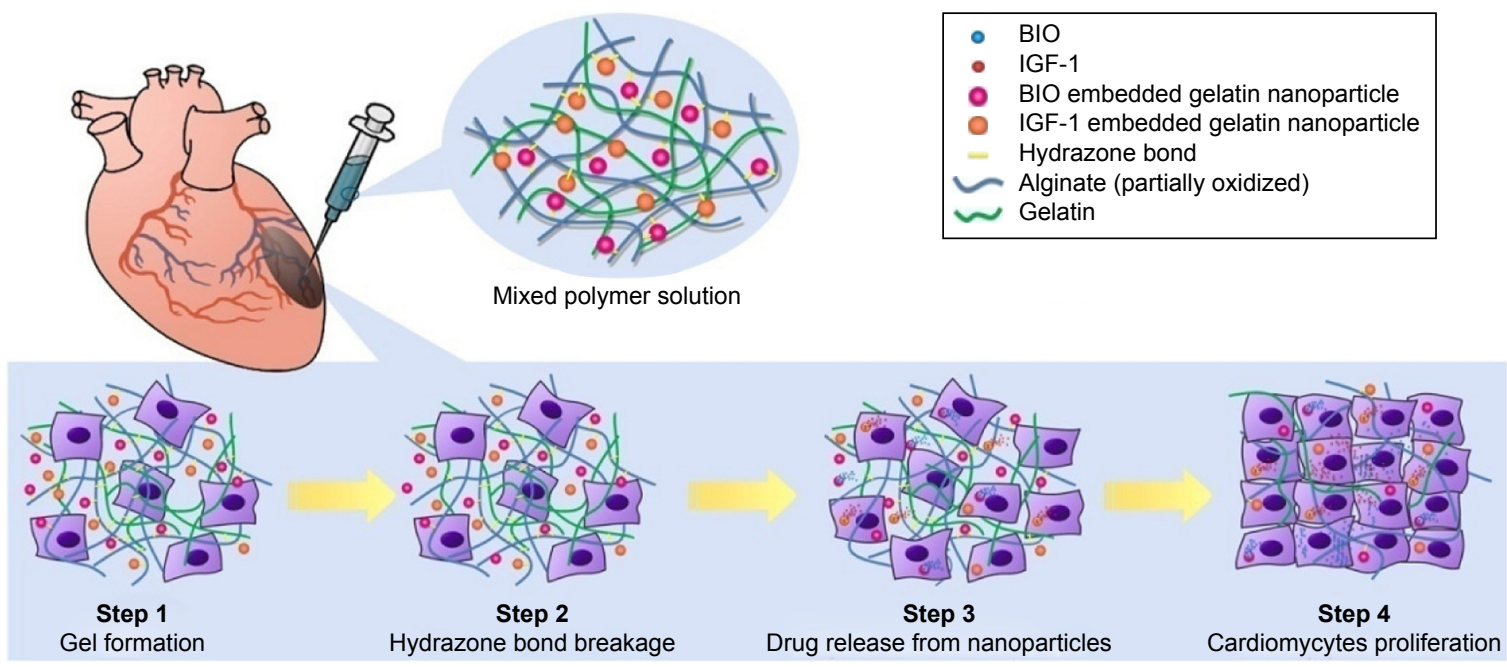

Figure 7 Scheme showing the mechanism of BIO release within the hydrogel after injection into the MI area.

Abbreviations: BIO, 6-bromoindirubin-3-oxime; IGF-I, insulin-like growth factor I; MI, myocardial infarction. 
the substrate, the NPs diffused quickly following injection into the infarcted area. Also, it was found that the fluorescence intensities decreased quickly at first, and then they slowly continued to decrease with the treatment of MI via the injection of hydrogels; this result may be due to the fact that our system is $\mathrm{pH}$ sensitive. Our results on the elastic and viscous moduli of the hydrogel illustrate that there is not much difference prior to and after release, which indicates that the hydrogel still maintains a gel network structure. As such, the controlled release profile was mainly caused by the breakdown of the chemical bond between NPs and hydrogel, rather than by the drug itself.

\section{Heart regeneration with our novel hybrid hydrogel system of chemically encapsulated gelatin NPs}

With the development of intramyocardial biomaterial, more and more biomaterials are being used for injection therapy. ${ }^{29}$ To date, it has already been demonstrated that many small molecules contribute to heart regeneration; however, the mechanisms by which these benefits are obtained remain unclear. ${ }^{34}$ Here, we show that following BIO administration, the number of resident cardiac cells in an MI model increased. Our findings further indicate that the function of BIO can promote dedifferentiation and proliferation in terminally differentiated cardiomyocytes in vivo. The BIO could reprogram the cardiomyocytes with changed morphology evidenced through rearrangement of the cytoskeleton, ${ }^{15}$ which can be clearly observed in the anticardiac troponin-T staining in Figure 5. In a recent study by Kohler et al it was demonstrated that low doses of BIO also have the ability to induce partial dedifferentiation among endothelial cells by regulating the canonical Wnt pathway. ${ }^{35}$ A previous study has shown that there is a dual role for BIO, which involves the maintenance of stem cell properties and the induction of proliferation in differentiated cardiomyocytes; this indicates that these functions may share common molecular pathways that control the canonical Wnt pathway. ${ }^{15,36}$

Secondly, our present findings document that IGF-1 can induce angiogenesis following MI. Consistent with our study, a previous study showed that the local delivery of the IGF-1 gene by recombinant adeno-associated virus in the setting of acute MI resulted in sustained IGF-1 expression, increased angiogenesis, and improved cardiac function. ${ }^{37}$ Another study also showed that IGF-1 enhanced wound healing and induced angiogenesis via a vascular endothelial growth factor-independent pathway. ${ }^{38}$

A previous study showed that immobilizing IGF-1 with hydrogel could serve as a controlled drug-release platform, and that it could also enhance cardiac progenitor cell survival and differentiation. In a study on chronically infarcted pig hearts, IGF-1 and hepatocyte growth factor therapy also resulted in improved cardiac function when using hydrogels. ${ }^{30}$ In our current research, we developed a novel hybrid hydrogel system with chemically encapsulated gelatin NPs for the sustained co-delivery of BIO and IGF-1 in MI treatment. To our knowledge, this is the first report that remedies MI via the co-delivery BIO and IGF-1, which have been identified as potential candidates to guide postnatal stem progenitor cells toward a cardiomyogenic fate. ${ }^{13}$ In the present study, we demonstrated that the co-delivery of BIO and IGF-1 can significantly improve heart function, while promoting resident cardiomyocyte proliferation and revascularization. Therefore, both $\mathrm{BIO}$ and IGF-1 can be involved in contributing to the heart's functional recovery. As such, the entire study protocol employed in the present work is clinically feasible and may be performed in a conventional catheterization laboratory.

\section{Limitations}

The concentrations of $\mathrm{BIO}$ and IGF-1 that were used in the current work were adopted and used as per previous studies. ${ }^{15,22}$ In the present study, the co-delivery of these two agents promoted the function and regeneration of the heart following MI. However, it is not clear if the optimal concentration of the co-delivery of BIO and IGF-1 can play an even more significant role in improving heart function after MI, which would be interesting to pursue in the future. In addition, although experimental work on the in vivo drug release of NPs encapsulated in hydrogel showed a 45-day release for $\mathrm{Cy} 7$, we can't detect the release efficiency of BIO and IGF-1 directly. It would be interesting to observe the delivery of these drugs over a longer period of time. This line of inquiry also suggests that more non-invasive methods should be developed for drug tracing in the future.

\section{Conclusion}

A novel hydrogel delivery system was developed to deliver drugs specifically to the site of an MI to selectively enhance the proliferation of resident cardiac cells, resulting in enhanced heart function recovery. Our results showed that NPs covalently entrapped in hydrogel hold great promise for treating MIs and other related diseases given their ability to deliver a wide range of therapeutics.

\section{Acknowledgments}

This research is supported by the National Natural Science Foundation of China (grant 81361128005), the Fundamental 
Research Funds for the Central Universities (grant HIT. IBRSEM. 201334), the Opening Foundation of the State Key Laboratory of Cancer Biology (grant CBSKL201106), and the Opening Foundation of the State Key Laboratory of Space Medicine (grant SFA10K02). English language editing of this manuscript was provided by Journal Prep (Montreal, Quebec, Canada).

\section{Disclosure}

The authors report no conflicts of interest in this work.

\section{References}

1. Leri A, Kajstura J, Anversa P. Mechanisms of myocardial regeneration. Trends Cardiovasc Med. 2011;21:52-58.

2. Jopling C, Sleep E, Raya M, Martí M, Raya A, Izpisúa Belmonte JC. Zebrafish heart regeneration occurs by cardiomyocyte dedifferentiation and proliferation. Nature. 2010;464:606-609.

3. Smith AJ, Lewis FC, Aquila I, et al. Isolation and characterization of resident endogenous c-Kit+ cardiac stem cells from the adult mouse and rat heart. Nat Protoc. 2014;9:1662-1681.

4. Moran AE, Oliver JT, Mirzaie M, et al. Assessing the global burden of ischemic heart disease: part 1: methods for a systematic review of the global epidemiology of ischemic heart disease in 1990 and 2010. Glob Heart. 2012;7:315-329.

5. Go AS, Mozaffarian D, Roger VL, et al; American Heart Association Statistics Committee and Stroke Statistics Subcommittee. Executive summary: heart disease and stroke statistics - 2014 update: a report from the American Heart Association. Circulation. 2014;129:399-410.

6. Wang CC, Chen CH, Lin WW, et al. Direct intramyocardial injection of mesenchymal stem cell sheet fragments improves cardiac functions after infarction. Cardiovasc Res. 2008;77:515-524.

7. Lee ST, White AJ, Matsushita S, et al. Intramyocardial injection of autologous cardiospheres or cardiosphere-derived cells preserves function and minimizes adverse ventricular remodeling in pigs with heart failure post-myocardial infarction. J Am Coll Cardiol. 2011;57:455-465.

8. Sanganalmath SK, Bolli R. Cell therapy for heart failure: a comprehensive overview of experimental and clinical studies, current challenges, and future directions. Circ Res. 2013;113:810-834.

9. Shim W, Mehta A, Wong P, Chua T, Koh TH. Critical path in cardiac stem cell therapy: an update on cell delivery. Cytotherapy. 2013;15:399-415.

10. Wohrle J, von Scheidt F, Schauwecker P, et al. Impact of cell number and microvascular obstruction in patients with bone-marrow derived cell therapy: final results from the randomized, double-blind, placebo controlled intracoronary Stem Cell therapy in patients with Acute Myocardial Infarction (SCAMI) trial. Clin Res Cardiol. 2013;102:765-770.

11. Bersell K, Arab S, Haring B, Kühn B. Neuregulin1/ErbB4 signaling induces cardiomyocyte proliferation and repair of heart injury. Cell. 2009;138:257-270.

12. Heallen T, Zhang M, Wang J, et al. Hippo pathway inhibits Wnt signaling to restrain cardiomyocyte proliferation and heart size. Science. 2011;332:458-461.

13. Xin M, Kim Y, Sutherland LB, et al. Regulation of insulin-like growth factor signaling by Yap governs cardiomyocyte proliferation and embryonic heart size. Sci Signal. 2011;4:ra70.

14. von Gise A, Lin Z, Schlegelmilch K, et al. YAP1, the nuclear target of Hippo signaling, stimulates heart growth through cardiomyocyte proliferation but not hypertrophy. Proc Natl Acad Sci U S A. 2012;109:2394-2399.

15. Tseng AS, Engel FB, Keating MT. The GSK-3 inhibitor BIO promotes proliferation in mammalian cardiomyocytes. Chem Biol. 2006;13: 957-963.

16. Leri A, Rota M, Hosoda T, Goichberg P, Anversa P. Cardiac stem cell niches. Stem Cell Res. 2014;13:631-646.
17. Ungvari Z, Csiszar A. The emerging role of IGF-1 deficiency in cardiovascular aging: recent advances. J Gerontol A Biol Sci Med Sci. 2012;67: 599-610

18. Wang J, Zhou J, Powell-Braxton L, Bondy C. Effects of Igf1 gene deletion on postnatal growth patterns. Endocrinology. 1999;140: 3391-3394.

19. Li Q, Li B, Wang X, et al. Overexpression of insulin-like growth factor-1 in mice protects from myocyte death after infarction, attenuating ventricular dilation, wall stress, and cardiac hypertrophy. J Clin Invest. 1997;100:19911999.

20. Torella D, Rota M, Nurzynska D, et al. Cardiac stem cell and myocyte aging, heart failure, and insulin-like growth factor-1 overexpression. Circ Res. 2004;94:514-524.

21. Wang H, Shi J, Wang Y, et al. Promotion of cardiac differentiation of brown adipose derived stem cells by chitosan hydrogel for repair after myocardial infarction. Biomaterials. 2014;35:3986-3998.

22. Ruvinov E, Leor J, Cohen $\mathrm{S}$. The promotion of myocardial repair by the sequential delivery of IGF-1 and HGF from an injectable alginate biomaterial in a model of acute myocardial infarction. Biomaterials. 2011;32: $565-578$.

23. Kumar S, Boehm J, Lee JC. p38 MAP kinases: key signalling molecules as therapeutic targets for inflammatory diseases. Nat Rev Drug Discov. 2003;2:717-726.

24. Azarmi S, Huang Y, Chen H, et al. Optimization of a two-step desolvation method for preparing gelatin nanoparticles and cell uptake studies in 143B osteosarcoma cancer cells. J Pharm Pharm Sci. 2006;9: $124-132$.

25. Coester CJ, Langer K, van Briesen H, Kreuter J. Gelatin nanoparticles by two step desolvation - a new preparation method, surface modifications and cell uptake. J Microencapsul. 2000;17:187-193.

26. Bai X, Fang R, Zhang S, et al. Self-cross-linkable hydrogels composed of partially oxidized alginate and gelatin for myocardial infarction repair. J Bioact Compat Pol. 2013;28:126-140.

27. Nunn C, Zou MX, Sobiesiak AJ, Roy AA, Kirshenbaum LA, Chidiac P. RGS2 inhibits beta-adrenergic receptor-induced cardiomyocyte hypertrophy. Cell Signal. 2010;22:1231-1239.

28. Lee EJ, Khan SA, Park JK, Lim KH. Studies on the characteristics of drug-loaded gelatin nanoparticles prepared by nanoprecipitation. Bioprocess Biosyst Eng. 2012;35:297-307.

29. Nelson DM, Hashizume R, Yoshizumi T, Blakney AK, MaZ, Wagner WR. Intramyocardial injection of a synthetic hydrogel with delivery of bFGF and IGF1 in a rat model of ischemic cardiomyopathy. Biomacromolecules. 2014;15:1-11.

30. Koudstaal S, Bastings MM, Feyen DA, et al. Sustained delivery of insulin-like growth factor-1/hepatocyte growth factor stimulates endogenous cardiac repair in the chronic infarcted pig heart. $J$ Cardiovasc Transl Res. 2014;7:232-241.

31. Weiss VU, Lehner A, Kerul L, et al. Characterization of cross-linked gelatin nanoparticles by electrophoretic techniques in the liquid and the gas phase. Electrophoresis. 2013;34:3267-3276.

32. Edmondson R, Broglie JJ, Adcock AF, Yang. Three-dimensional cell culture systems and their applications in drug discovery and cell-based biosensors. Assay Drug Dev Technol. 2014;12:207-218.

33. Artzi N, Oliva N, Puron C, et al. In vivo and in vitro tracking of erosion in biodegradable materials using non-invasive fluorescence imaging. Nat Mater. 2011;10:704-709.

34. Cho J, Zhai P, Maejima Y, Sadoshima J. Myocardial injection with GSK-3 $\beta$-overexpressing bone marrow-derived mesenchymal stem cells attenuates cardiac dysfunction after myocardial infarction. Circ Res. 2011;108:478-489.

35. Kohler EE, Baruah J, Urao N, et al. Low-dose 6-bromoindirubin-3'oxime induces partial dedifferentiation of endothelial cells to promote increased neovascularization. Stem Cells. 2014;32:1538-1552.

36. Sato N, Meijer L, Skaltsounis L, Greengard P, Brivanlou AH. Maintenance of pluripotency in human and mouse embryonic stem cells through activation of Wnt signaling by a pharmacological GSK-3specific inhibitor. Nat Med. 2004;10:55-63. 
37. Dobrucki LW, Tsutsumi Y, Kalinowski L, et al. Analysis of angiogenesis induced by local IGF-1 expression after myocardial infarction using microSPECT-CT imaging. J Mol Cell Cardiol. 2010;48:1071-1079.
38. Balaji S, LeSaint M, Bhattacharya SS, et al. Adenoviral-mediated gene transfer of insulin-like growth factor 1 enhances wound healing and induces angiogenesis. J Surg Res. 2014;190:367-377.

\section{Publish your work in this journal}

The International Journal of Nanomedicine is an international, peerreviewed journal focusing on the application of nanotechnology in diagnostics, therapeutics, and drug delivery systems throughou the biomedical field. This journal is indexed on PubMed Central, MedLine, CAS, SciSearch $\AA$, Current Contents ${ }^{\circledR} /$ Clinical Medicine,
Journal Citation Reports/Science Edition, EMBase, Scopus and the Elsevier Bibliographic databases. The manuscript management system is completely online and includes a very quick and fair peer-review system, which is all easy to use. Visit http://www.dovepress.com/ testimonials.php to read real quotes from published authors.

Submit your manuscript here: http://www.dovepress.com/international-journal-of-nanomedicine-journal 